\title{
El cuerpo sexuado, el sombrero con pluma y el automóvil. Phénoménologie de la perception leída desde una perspectiva feminista y performativa
}

Recibido el 15/og/2020. Aceptado el 30/11/2020.

\begin{abstract}
Resumen
Este trabajo presenta la teoría merleau-pontyana de la sexualidad contenida en su Phénoménologie de la perception, primeramente en el contexto de otros tempranos abordajes fenomenológicos de la misma cuestión y luego dentro del marco gnoseológico de la obra. A continuación, se evalúa el alcance de las primeras críticas que J. Butler realizó a tal teoría en términos de androcentrismo, naturalización de la diferencia sexual y de la dominación masculina. Se deslindan así los recursos y los límites que comporta la descripción merleau-pontyana del cuerpo sexuado como "expresión" y "estilo corporal" para la elaboración de una concepción no esencialista de la sexualidad afín a la teoría performativa.
\end{abstract}

Palabras clave: Merleau-Ponty, Butler, Sexualidad, Feminismo, Fenomenología.

The Sexed Body, the Plumed Hat, and the Automobile. Phénoménologie de la Perception Read from a Feminist and Performative Perspective

\begin{abstract}
This paper presents the Merleau-Pontyan theory of sexuality contained in his Phénoménologie de la perception, firstly in the context of other early Phenomenological approaches to the same topic, and then within the gnoseological framework of the work. The scope of J. Butler's early criticisms of such a theory in terms of androcentrism, naturalization of sexual difference and male dominance is then evaluated. By this means, it becomes possible to define the resources and limits of the Merleau-Pontyan description of the sexed body as "expression" and "body style" for the elaboration of a non-essentialist conception of sexuality akin to performative theory.
\end{abstract}


La Phénoménologie de la perception de Merleau-Ponty propone, desde su título, abordar un problema específico de la teoría del conocimiento desde el marco de una corriente filosófica particular. Sin embargo, lo que la obra de hecho ofrece desborda por mucho ese estrecho encuadre: a lo largo de sus páginas se halla formulada una concepción filosóficamente inédita de la experiencia corporal en sus múltiples dimensiones, incluida la sexualidad en su integración a "todo el ser cognoscente y agente" (Merleau-Ponty, 1967: 184). El capítulo "El cuerpo como ser sexuado" (180202), dedicado íntegramente a esta última cuestión, fue objeto de una crítica minuciosa por parte de J. Butler en dos tempranos ensayos de la década de los ' $80(1988,1989)^{2}$ lo que sugiere que la lectura que la filósofa realizó de la fenomenología merleaupontyana del cuerpo jugó un rol importante en la gestación de su propia teoría performativa del género y la sexualidad. ${ }^{3}$ Si bien en estos dos trabajos Butler se detiene más bien en los desaciertos, prejuicios e insuficiencias que percibe en los análisis de Merleau-Ponty y formula su propia teoría performativa en franca oposición a la teoría fenomenológica de este último, propondré en estas páginas que la propuesta merleau-pontyana, lejos de ser incompatible con la alternativa propuesta por Butler en estos ensayos, más bien y por el contrario aporta valiosos recursos conceptuales para una teoría no esencialista ni naturalista de la sexualidad como la que Butler y otrxs teóricxs feministas han desarrollado en las últimas décadas. ${ }^{4}$ Si Butler estima que la teoría feminista tiene "algo que ganar" pero sobre todo "algo que temer de la teoría de la sexualidad de Merleau-Ponty" (Butler, 1989: 86), el propósito central de este trabajo será entonces mostrar que la teoría feminista tiene "más que ganar" de la fenomenología merleau-pontyana que lo que Butler advirtió. Sin embargo, como señalaré al final, considero que esto no invalida en absoluto las críticas formuladas por la filósofa, puesto que solo a través suyo y de las diversas relecturas y revisiones feministas posteriores la Phénoménologie reveló y sigue revelando tanto sus límites como sus recursos para pensar la sexualidad en el marco de los nuevos desafíos de este siglo.'

Al enfocar esta indagación en la teoría de la sexualidad esbozada en Phénoménologie y en las tempranas críticas que Butler realizó puntualmente a tal teoría, estas consideraciones comportarán ciertas limitaciones bibliográficas y temáticas. Con el fin de priorizar las afinidades y desajustes entre los particulares modos en que cada filósofx comprende la sexualidad como "estilo corporal" y como "expresión" dejaré de lado aquí la interesante discusión, ya abordada en estudios previos de diversxs autorxs, en torno a las ventajas que la perspectiva merleau-pontyana centrada en el cuerpo y en la experiencia vivida podría acarrear respecto del acento lingüístico que Butler frecuentemente imprime a su teoría performativa (cfr. por ejemplo Alcoff, 2000; Bordo, 1998; Bourdieu, 1998: 81; García, 2012). Por otro lado, con el objeto de poner el foco en las ideas propuestas en Phénoménologie, haré a un lado aquí las referencias de Butler a

\footnotetext{
1 Agradezco a Danila Suárez Tomé y Milton Abellón, como así también a lxs evaluadorxs anónimos, por su atenta lectura y sus valiosos comentarios acerca de los muchos puntos discutibles de la interpretación que presento en este trabajo.

2 Como aclara Butler en nota al pie, el ensayo publicado en 1989 fue originalmente redactado en 1981 (1989: 85).

3 Me referiré aquí en general a la "sexualidad" abarcando las diferentes cuestiones de la identidad sexo-genérica y de la orientación sexual o el deseo, haciendo explíitito cuando me refiera específicamente a una u otra cuestión. Además, utilizaré los términos sexo y género de modo intercambiable teniendo en cuenta, no solo el uso butleriano de los términos en estos tempranos ensayos, sino el hecho de que su teoría performativa conduce en escritos posteriores a una crítica explícita de tal distinción (cfr. Butler, 1990: 8-10). Por otra parte, como señala acertadamente Foultier y este trabajo confirmará en cierto modo, puede sostenerse también que "la crítica de Merleau-Ponty al pensamiento objetivo [...] provee un marco que rompe y apunta más allá de la oposición entre sexo y género, [...] naturaleza y cultura" (Foultier, 2013: 778). 4 El uso lingüístico del género masculino como universal será objeto de discusión en este trabajo. Adopto aquí el lenguaje inclusivo en consonancia con el art. 1 de la Resolución del 9/12/2919 del Consejo Directivo de la Facultad de Filosofía y Letras de la Universidad de Buenos Aires que reconoce su validez en las producciones académicas. Me refiero aquí a las "teorías feministas" en el más amplio sentido del término, como se advertirá en las referencias ulteriores, abarcando diversas vertientes de tal corriente -y obviando para el propósito circunstancial de estos análisis sus tensiones internas-, en sintonía con la definición abierta a la que Butler suscribe en el cap. 1 de Vidas en lucha. Conversaciones (2019).

5 En este aspecto, este trabajo coincide con la conclusión de Foultier (2013), si bien arriba a ella por una vía diferente. A diferencia de la autora, quien considera que el capítulo en cuestión de Phénoménologie no propone "una teoría de la sexualidad" (778), sostendré aquí, más cerca de Butler y a diferencia de Foultier, que una tal teoría está de hecho allí en juego, si bien no precisamente la que Butler cree encontrar. Este trabajo es también deudor de los análisis comparativos previos de Oksala (2006) y Stoller (2010) que serán citados infra.
} 
textos posteriores del filósofo, y en particular sus sugestivos comentarios en torno a Le visible et linvisible, obra en la que ve esbozada una alternativa teórica distinta de la que es objeto de su crítica (cfr. Butler, 2006a: 193-194; 2006b; 1989: 99-100, n. 18; 2011:88).

\section{La fenomenología del sexo y el sexo de lxs fenomenólogxs}

Si bien Husserl incorporó muy tempranamente a su investigación fenomenológica como una cuestión de máxima importancia la del Leib (el "cuerpo viviente" o "propio"), la sexualidad como tal comenzó a ser mencionada como tema propio de su reflexión solo después de 1930, al anexar a sus previas descripciones estáticas y genéticas una tercera dimensión "generativa”. Ésta abarcaría, más allá del pasado personal sedimentado en habitualidades, "un horizonte más amplio del pasado que no está a la medida del recuerdo [...] en tanto generado por padres, nacido en el mundo" (Hua XV: 580), lo que lo condujo a considerar simultáneamente la posibilidad de "una conciencia instintiva y una comunicación instintiva" (Hua XV: 608). Así, en su Krisis menciona "el problema de los sexos" (Hua VI: 192), y en manuscritos publicados póstumamente puede leerse: "Este apetito sexual determinado tiene la forma de la satisfacción en el modo de la copulación" (Hua XV: 593). Ciertamente, sus observaciones acerca del tema pueden resultar taciturnas o lacónicas, como señalan J. Oksala (2004: 16) o S. Heinämaa (2003: 53), pudiendo resumirse, de acuerdo con F. A. Elliston (1981: 333), en la tesis de que el sexo es un acto social, heterosexual y que apunta a la copulación. Sin embargo, Merleau-Ponty advirtió refiriéndose a "los Inéditos" que estas tímidas y controvertibles definiciones encerraban a la vez una cierta osadía: en ellas, en un gesto inaugural para la fenomenología y singular en el contexto más amplio de la tradición filosófica, afirma Merleau-Ponty, "se considera el instinto sexual 'desde el punto de vista trascendental", lo que apunta, en su visión, a una profunda "reforma de la conciencia" en la que "las estructuras de la afectividad sean constituyentes ni más ni menos que las otras" (1964: 291-292).

La fenomenología francesa recogió este desafío del último Husserl como no lo hizo, en cambio, Heidegger, quien paradójicamente pretendía deshacerse del presunto intelectualismo husserliano bajándolo al suelo de la existencia concreta. Como observa Sartre en 1943, Heidegger "no alude para nada a ella [la sexualidad] en su analítica existencial, de suerte que su 'Dasein' nos aparece como asexuado" (1943: 423). El Dasein es sin sexo, señala Sartre, no solo en tanto la existencia es ajena al deseo sino también a la diferencia sexual: "el hombre, como la mujer, 'existe', ni más ni menos" (Sartre, 1943: 423), aunque puede observarse que en la analítica heideggeriana el Dasein adopta fisonomías semejantes a las del operario y el héroe, asociadas fuertemente en nuestra cultura patriarcal con la virilidad (cfr. Palacio, 2011: 673). Ciertamente, Lêtre et le néant se refiere en cambio al hombre y a la mujer, pero a esta última exclusivamente como objeto del amor, del deseo o de la visión del primero: la mujer es, en suma "el otro deseado" (1943: 435) o "el objeto deseado" (423): "cada objeto poseído que se alza sobre el fondo de mundo manifiesta el mundo entero, como la mujer amada manifiesta el cielo, la playa, el mar que la rodean" (642). La obra ofrece también vívidas descripciones fenomenológicas de los carices más frustrantes y patéticos de la experiencia erótica: la voluptuosidad es "turbación", el deseo es la elección de la conciencia de hacerse carne para apropiarse de la carne del otro, y finalmente

6 Merleau-Ponty se refiere particularmente al escrito de Husserl titulado "Universale Teleologie" de 1933, luego publicado en Hua XV (593612).

7 La mujer como sujetx de la acción o de la mirada solo es considerada desde el punto de vista de su instrumentalización por parte del hombre masoquista, o de la vergüenza del "nosotros" masculino por ella mirado $(1943: 419,455)$, vale decir, solo desde el punto de vista masculino. 
el placer del orgasmo es el fracaso de ese proyecto cifrado en el deseo (401-453). Así, la insatisfacción, la culpabilidad y la impotencia no designan, en la visión sartriana, ciertas posibilidades particulares a la que está abierta la experiencia sexual, sino que definen su esencia, haciendo patente a su vez de modo emblemático la intrínseca frustración que caracterizaría a la totalidad de nuestras relaciones posibles con lxs otrxs.

Dos años más tarde, en 1945, Merleau-Ponty retomó la tarea de desarrollar una fenomenología de la experiencia sexual, incluyendo en su Phénoménologie un capítulo de tono menos dramático y con descripciones menos gráficas que las que caracterizaban las páginas de Sartre sobre el tema. En una primera lectura, estas diferencias de estilo redundan en la impresión de un enfoque menos atormentado pero, a la vez, "menos sexual" de la sexualidad que el propio de su amigo y precursor en este terreno: las referencias más específicas a la cuestión, al principio del capítulo, se diluyen tras las pocas primeras páginas en consideraciones más abstractas acerca del psicoanálisis existencial y el cuerpo como "expresión de la existencia", nexo entre lo voluntario y lo involuntario, la conciencia y el automatismo. Más allá de estos contrastes generales de tono y estilo, Phénoménologie adopta en algunos pasajes la misma analogía que recorría las páginas de L'être et le néant entre la mujer y el objeto poseído: "Paul sufre porque ha perdido a su mujer o se enoja porque le han robado el reloj" (Merleau-Ponty, 1967: 409). ${ }^{8}$ Antes de detenernos en el capítulo en cuestión de Phénoménologie es necesario mencionar, en esta acotada semblanza de algunos de los primeros encuentros de la fenomenología y la sexualidad, que en la misma década E. Levinas publica $D e$ l'existence à l'existant (1947) donde afirma que "el otro por excelencia es lo femenino" (1990: 145), una tesis que reitera en sus conferencias de 1947-1948 publicadas como "Le temps et l'autre".' Inmediatamente, en Le deuxième sexe (1949), Simone de Beauvoir se refiere críticamente a estas afirmaciones señalando su sesgo androcéntrico:

Supongo que el señor Levinas no olvida que la mujer es también, para sí, conciencia. Pero es chocante que adopte deliberadamente un punto de vista de hombre, sin señalar la reciprocidad del sujeto y del objeto. Cuando escribe que la mujer es misterio, sobreentiende que es misterio para el hombre. De modo que esta descripción que se pretende objetiva, es de hecho una afirmación del privilegio masculino (Beauvoir, 1976a: 20, n.10).

Como lo mostraría la teorización feminista posterior, estos señalamientos de Beauvoir permitían ser extendidos retroactivamente a las previas consideraciones sartrianas y merleau-pontyanas sobre el tema, e inaugurarían maneras radicalmente nuevas en que los estudios fenomenológicos y la sexualidad se entrelazarían durante las décadas siguientes. Al recordar sus primeras lecturas del existencialismo francés en su Prefacio a Gender Trouble (1990), Butler observó que si su lectura de Beauvoir le enseñó que ser mujer en una cultura masculinista significa ser un "problema" y un "misterio", pudo hallar una confirmación concreta de la tesis beauvoiriana al leer a Sartre, "para quien todo deseo, problemáticamente supuesto como heterosexual y masculino, se definía como problema (trouble)" (Butler, 2006: xxix, xxx).

\footnotetext{
8 Así como "Pierre" era el "protagonista filosófico" de L'imaginaire (1940), las referencias a "Pierre" y "Paul" atraviesan L'être et le néant, siendo características del estilo de redacción sartriano (si bien no exclusivamente). Al adoptar este estilo ocasionalmente en su obra, MerleauPonty connota usualmente una referencia a ideas o temas de su compañero.

9 Ciertamente, las cuestiones del erotismo y de la diferencia sexual no pueden reducirse en Levinas a lo que puede sugerir esta escueta referencia, como muestra Palacio (2001). Para una visión más amplia puede consultarse asimismo el volumen Feminist Interpretations of Levinas (Chanter (Ed.), 2001).

10 Butler analiza extensamente la concepción sartriana del deseo en el capítulo III de Subjects of Desire (1987). Para una crítica de la lectura butleriana de Sartre cfr. Bolla (2015).
} 


\section{2. "El cuerpo como ser sexuado" en el marco de Phénoménologie de la perception}

En el contexto de esta década de 1940, tan atribulada histórica y políticamente y a la vez tan fecunda para la fenomenología en Francia, Merleau-Ponty propone en su Phénoménologie de la perception comprender la percepción como una función del cuerpo. A lo largo de sus páginas, Phénoménologie va dando forma a esta apuesta teórica original dialogando críticamente con las posiciones de muy diversos interlocutores filosóficos y científicos: si el título anuncia una "fenomenología", las reflexiones que se desenvuelven en la obra desde el "Avant-Propos" -que se pregunta problemáticamente "qué es la fenomenología"- suponen más bien una reformulación del método fenomenológico mismo, sosteniendo un diálogo horizontal no solo con otras corrientes filosóficas sino con ciencias humanas y naturales, e incluso con obras literarias. Proponer que la percepción tiene su sede en el cuerpo significa oponerse a las teorías -por ejemplo, de cuño kantiano- que la consideran dependiente de funciones intelectuales, pero significa también redescubrir el cuerpo que percibe, el cual no se reduce al ensamble de órganos y funciones descripto por las ciencias objetivas. Es así que, en los primeros cuatro capítulos de la Primera Parte de la obra (titulada "El cuerpo"), para cumplir este propósito de redescubrimiento Merleau-Ponty integra su "voluptuosa" lectura de las descripciones husserlianas del Leib en Ideen II ${ }^{11}$ con resultados empíricos de la psicología y la neurología, desbaratando los marcos científicos clásicos que restringen nuestra concepción del cuerpo y sus capacidades (Merleau-Ponty, 1967: 87-179). En la experiencia tal como la vivimos no tenemos conciencia de nuestro cuerpo en términos de una imagen o representación psíquica, ni tampoco como el agregado anátomo-fisiológico descripto por las ciencias: contamos, en cambio, con una conciencia corporal del cuerpo -nuestro cuerpo sabe de sí mismo- ${ }^{12}$ como estando en tal posición y tal situación pragmática, con determinadas posibilidades de acción y comportamiento ofrecidas por un entorno que es percibido, a su vez, en correlación con nuestras disposiciones motrices: "tengo conciencia de mi cuerpo a través del mundo [...] [y] tengo conciencia del mundo por medio de mi cuerpo" (Merleau-Ponty, 1967: 97). Este "esquema corporal", un repertorio de capacidades prácticas latentes que regula nuestra propiocepción y correlativamente la percepción del mundo, es definido también como un "cuerpo habitual", en tanto se constituyó (y continúa haciéndolo) en función de la sedimentación de hábitos motrices: la adquisición de un hábito es siempre una "reorganización y renovación del esquema corpóreo" (1967: 166). Mediante el examen de diversos ejemplos como los del caminar, el bailar, el uso de vehículos como un automóvil, de útiles y de máquinas, de instrumentos musicales o de indumentaria y accesorios como un sombrero con pluma, Merleau-Ponty muestra que la plasticidad y transponibilidad característica de estas habitualidades las distingue de los automatismos reflejos, retirándolas así del ámbito puramente fisiológico, pero su adquisición y ejercicio hacen patente también que no resultan de la intervención de procesos intelectuales: sale a la luz de este modo "un saber que está en las manos" (1967: 168) o, más generalmente, "un cuerpo que comprende".

Desde estas premisas concernientes al esquema corporal y al hábito establecidas en los primeros capítulos Merleau-Ponty se propone, en el quinto de ellos, abordar "El cuerpo como ser sexuado". Tal como lo adelantamos, el autor solo dedica las primeras páginas, escasas pero altamente sugestivas, a exponer una perspectiva

11 La lectura de los manuscritos entonces inéditos de Ideen II (en Louvain, 1939) provocó a Merleau-Ponty, en sus palabras, "una experiencia casi voluptuosa" (cfr. Rojcewicz-Schuwer, 1989: xvi).

12 "Si se puede hablar todavía en la percepción del cuerpo propio de una interpretación será necesario decir que el cuerpo se interpreta a sí mismo" (Merleau-Ponty, 1967: 175).

13 “Mi cuerpo tiene su mundo o comprende su mundo sin tener que pasar por unas 'representaciones'” (Merleau-Ponty, 1967: 164). 
"fenomenológica corporal" de la sexualidad per se, mientras que el resto de su análisis se detiene más largamente en la relación de la sexualidad con otras dimensiones, "corrientes" o "sectores" de la existencia, con "todo el ser cognoscente y agente" o, en fin, con "la existencia" en general (Merleau-Ponty, 1967: 184 ss.). Si ya no cabe concebir una conciencia incorporal y la organización corporal no es "un hecho contingente" del ser humano total distinguible de sus predicados necesarios (198), entonces la fenomenología y la filosofía en general no pueden obviar, como se hizo tradicionalmente, la dimensión sexual de la existencia: "un hombre sin manos o sin sistema sexual es tan inconcebible como un hombre sin pensamiento" (198). De acuerdo con Merleau-Ponty, la integración de esta dimensión menoscabada a los demás aspectos más decorosos de la existencia humana se realizó, por ejemplo y primeramente, en el psicoanálisis de $\mathrm{S}$. Freud, y de diversos otros modos en la ontología fenomenológica de J.-P. Sartre o la psicología existencial de L. Binswanger, tres referentes que atraviesan el entero capítulo. Merleau-Ponty encuentra en cada una de estas vertientes la misma valiosa enseñanza que hace propia, a saber, que entre la sexualidad y la existencia se traba una relación de "expresión" recíproca, en un sentido particular de "expresión” que será necesario precisar más adelante. Así, recoge, comenta y reivindica las ideas de estos autores en la medida en que se acomodan a esta interpretación, pero advierte que sus propuestas se exponen a "dos errores" que es necesario evitar (196). Por un lado, existe el riesgo existencialista de diluir el carácter propiamente corporal y sexual de la sexualidad para ver en ella solamente el símbolo de las alternativas metafísicas más generales de la existencia humana. Por otro lado, el freudismo se arriesga a concebir la sexualidad en términos de "representaciones inconscientes" que dominan secretamente la existencia. En cambio, afirma MerleauPonty, "la sexualidad no es trascendida en la vida humana ni figurada en su centro por representaciones inconscientes. Está constantemente presente en ella como una atmósfera", como "esa parte de nosotros mismos siempre somnolienta que sentimos por debajo de nuestras representaciones, esta bruma individual a través de la cual percibimos el mundo", y que "irradia" de nuestro cuerpo "como un olor o un sonido" (196). El reconocimiento de esta "ósmosis" o "difusión" de la sexualidad a todas las dimensiones de la existencia no impide, sino que, por el contrario, requiere que se defina la especificidad de la "zona sexual" de la existencia como "un modo original de conciencia" (181), a riesgo de que la sexualidad como tal pierda todo significado: aun si es "coextensiva" con la vida, no puede ser su sinónimo (197). Y es precisamente al procurar esta definición cuando Merleau-Ponty expone sus perspectivas más singulares y fecundas sobre la cuestión.

Los análisis de Steinfeld acerca del "paciente Schneider", quien habiendo sufrido una lesión occipital manifiesta una "inercia sexual", sirven a Merleau-Ponty para ilustrar que la sexualidad no depende única ni principalmente de partes o funciones orgánicas "sexuales", pero tampoco de representaciones mentales relativas al sexo. ${ }^{15} \mathrm{El}$ paciente cuenta con ambas, solo que ni las partes ni las representaciones están "animadas" por un valor o una significación erótica o libidinal: "el enfermo ha dejado de dirigir al contexto inmediato esta pregunta muda y permanente que es la sexualidad normal"(183). Así como en los capítulos previos de la obra el cuerpo tal como es vivido revelaba su enclave más propio en la dimensión del comportamiento, ontológicamente ambigua e irreductible a la materia y al espíritu, por vía del examen de la sexualidad se despeja una vez más este mismo ámbito original, el de una "intencionalidad corporal" que ahora es declarada también "libidinal". Si Merleau-Ponty resumía el modo en que nuestras disposiciones y capacidades motrices latentes nos 
hacen habitar un entorno significativo en la fórmula del "esquema corporal", ahora éste adquiere la connotación agregada de ser un "esquema sexual" dotado de una "percepción erótica" y una "comprensión erótica" (182-183):

Un espectáculo tiene para mí una significación sexual no cuando me represento, siquiera confusamente, su relación posible con los órganos sexuales o con los estados de placer, sino cuando existe para mi cuerpo, para esta potencia siempre lista para trabar los estímulos dados en una situación erótica y ajustar una conducta sexual a la misma. [...] El cuerpo visible está subtendido por un esquema sexual, estrictamente individual, que acentúa las zonas erógenas, dibuja una fisonomía sexual y reclama los gestos del cuerpo masculino, él mismo integrado a esta totalidad afectiva (182-183).

\section{Androcentrismo, naturalización y reificación de la dominación masculina en Phénoménologie}

En "Sexual Ideology and Phenomenological Description" (1989), un ensayo dedicado a criticar con detalle el capítulo de Phénoménologie acerca de la sexualidad, Butler observa perspicazmente que en el párrafo recién citado lxs lectorxs no sabemos hasta el final, donde se menciona el "cuerpo masculino", para quién son erógenas las zonas en cuestión, quién es $e l$ que ve y quién la que es vista. Esta y otras vacilaciones y eufemismos de la enunciación connotan, para Butler, una implícita universalización o esencialización, no solo del carácter heterosexual de la sexualidad sino de los roles del hombre como sujeto deseante y la mujer como objeto deseado, siendo éstos rasgos que luego Gender Trouble asociará a una "matriz heterosexual" que restringe nuestra comprensión de la sexualidad a términos binarios y jerárquicos. ${ }^{16}$ En la sección del mismo ensayo titulada "La misoginia como estructura intrínseca de la percepción" (Butler, 1989: 92-95) la filósofa afirma que en estos análisis merleau-pontyanos el sujeto masculino no solo proyecta "su propio deseo en el cuerpo femenino" sino que consagra tal proyección como "la estructura misma del cuerpo que percibe", cerrando el círculo solipsista del voyeur masculino (Butler, 1989: 94).

Esta primera línea de crítica podría ser matizada teniendo en cuenta, en primer lugar, que Merleau-Ponty se refiere frecuentemente al "hombre" con el significado (cuestionablemente) universal del "ser humano en general" reflejando los usos lingüísticos epocales. ${ }^{17}$ Merleau-Ponty los adopta al punto de que, si se leyese textualmente l'homme con su connotación masculina, debería pensarse -siguiendo la lectura literal de Butler- que concebía la sexualidad como privativamente homosexual: "Si la historia sexual de un hombre (sic) provee la clave de su vida, es porque en su sexualidad se proyecta su manera de ser respecto del mundo, es decir, respecto del tiempo y de los otros hombres (sic)" (Merleau-Ponty, 1967: 185). Pero es más relevante observar, en segundo lugar, que cuando Merleau-Ponty habla de la experiencia sexual adoptando el punto de vista de un sujeto masculino y heterosexual, está explicitando la posición sexuada particular del autor o bien del caso puntual del "paciente Schneider" que está de hecho analizando ( $c f r$. Foultier, 2013: 774). Esto podría considerarse no solo como justificado sino aun como necesario en el contexto de una descripción fenomenológica

16 Butler define la "matriz heterosexual" como "aquella grilla de inteligibilidad cultural por medio de la cual cuerpos, géneros y deseos son naturalizados. Me apoyo aquí en la noción de M. Wittig de 'contrato heterosexual' y, en menor medida, en la noción de A. Rich de 'heterosexualidad obligatoria' para caracterizar un modelo hegemónico discursivo/epistémico de inteligibilidad genérica que supone que para que los cuerpos sean coherentes y hagan sentido debe haber un sexo estable que se expresa a través de un género estable [...] que es definido oposicional y jerárquicamente a través de la práctica obligatoria de la heterosexualidad" (Butler, 2010: 7, n. 6).

17 Me expreso aquí en condicional ya que el alcance de estas líneas de "defensa" a Merleau-Ponty será relativizado en la última sección de este trabajo. 
de la experiencia. ${ }^{18}$ Ciertamente, Merleau-Ponty evita de este modo incurrir en la vía, indudablemente más objetable, de pretender desarrollar una fenomenología de la experiencia sexual universal -o femenina- desde una posición masculina no explicitada que presume de una falsa neutralidad o universalidad.

En el mismo ensayo Butler critica además la supuesta naturalización o "reificación" merleau-pontyana de la opresión del hombre sobre la mujer imperante en nuestra cultura sexista, al declarar "esencial" o "metafísico" el modelo de relación amoesclavo asignando el primer rol al varón y el segundo a la mujer (Butler, 1989: 86). Sin embargo, esta crítica también puede ser contrapesada de considerar, en primer lugar, el hecho cierto -e ignorado en el análisis de Butler- de que Merleau-Ponty está en este punto de su exposición reseñando los desarrollos de Sartre con el fin ulterior de relativizar su alcance, no solo por cuanto se arriesgan a diluir la sexualidad en la existencia, sino en la medida en que la señalada dialéctica no constituye para Merleau-Ponty la figura primaria ni el destino último de las relaciones interhumanas posibles (cfr. Merleau-Ponty, 1967: 398-419). ${ }^{19}$ En segundo lugar, debe tenerse en cuenta también que la "objetivación", la opresión u otras condiciones "metafísicas" o "esenciales" de la existencia son entendidas por Merleau-Ponty -tal como lo es su carácter "esencialmente corporal" y sexual-, como caracteres propios de la existencia humana en general. En los términos textuales del filósofo, tales figuras aluden a "una vivencia, dada a todos y siempre accesible, de la condición humana en sus momentos más generales de autonomía y de dependencia" (1967: 195). Si bien Butler afirma que Merleau-Ponty "conceptualiza la relación sexual entre hombres y mujeres bajo el modelo del amo y el esclavo" (1989: 86), no es en estos términos generizados e irreversibles como el filósofo efectivamente se expresa, sino que enuncia textualmente, en primera persona: "en cuanto tengo un cuerpo, puedo ser reducido a objeto bajo la mirada del otro y no contar ya para él como persona, o bien, al contrario, puedo pasar a ser su dueño y mirarlo a mi vez"(Merleau-Ponty, 1967: 194). Si Beauvoir no dirigió a Sartre ni a Merleau-Ponty la misma crítica que aplicó a Levinas, fue precisamente porque, a diferencia de Butler, comprendió los análisis de aquéllos en el sentido en que Merleau-Ponty explícitamente los formuló, es decir, como apuntando a condiciones o roles generales e intercambiables:

el hombre es, como la mujer, carne, y por tanto, pasividad [...] y ella, como él, [...] actividad; cada uno de ellos vive a su manera el extraño equívoco de la existencia hecha cuerpo. [...] En los dos sexos se desarrolla el mismo drama de la carne y el espíritu, de la finitud y la trascendencia; a ambos los roe el tiempo, lo acecha la muerte (Beauvoir, 1976b: 495-496).

Butler vuelve a referirse al capítulo de Phénoménologie sobre la sexualidad en "Performative Acts and Gender Constitution" (1988), cuando esboza por primera vez su teoría performativa eligiendo precisar su posición justamente por vía de una contraposición con una teoría "expresiva" de cuño "fenomenológico". ${ }^{20}$ Allí sostiene que una tal teoría, representada emblemáticamente por Merleau-Ponty, no puede evitar pensar lo masculino y lo femenino en términos naturalistas o esencialistas en la medida en que postula que el comportamiento corporal y sexual es una "expresión" de la existencia

\footnotetext{
18 Esta interpretación puede confirmarse al leer en continuidad la citada frase de Phénoménologie con la posterior descripción merleaupontyana de "una mujer" en Le langage indirect et les voix du silence-a la que volveré más adelante-, donde el filósofo comienza explicitando que su descripción se enmarca en su propia posición sexuada masculina: "Una mujer que pasa no es primeramente para mí un contorno corporal [...]" (Merleau-Ponty, 1960: 67). Este problema de la "objetividad subjetiva" en fenomenología y sus implicancias para una fenomenología feminista es puesto de relieve y analizado por L. Fisher (2000: 17-38), discutiendo también la lectura butleriana de Merleau-Ponty. 19 Si bien Butler reconoce las diferencias entre las teorías merleau-pontyana y sartriana de la percepción y el cuerpo, en Subjects of Desire parece no advertir las marcadas diferencias existentes entre sus concepciones de la intersubjetividad y la sexualidad, consignando solo que "la discusión de Sartre del deseo sexual [...] se acerca mucho a la de la Fenomenología de la percepción de Merleau-Ponty" (1987: 148). 20 Esta oposición se reiterará en Gender Trouble: "la distinción entre expresión y performatividad es crucial" (2010: 192).
} 
en general, y aun de un sujeto o una identidad que precede a sus expresiones. Esto se cumpliría indefectiblemente, ya sea que lxs fenomenólgxs supongan clásicamente (en la línea de Husserl) una "conciencia constituyente" y/o, en las versiones fenomenológicas más renovadas de tipo merleau-pontyano o beauvoiriano, cuenten con el bagaje de una dimensión "natural" del cuerpo que sería luego asumida de diversos modos en la vida personal. Los modelos fenomenológicos "asumen un yo antepuesto a sus actos" (Butler, 1988: 520), mientras que desde la perspectiva butleriana, en cambio, "el cuerpo adquiere su género en una serie de actos que son renovados, revisados y consolidados en el tiempo" (1988: 523), en el marco de convenciones culturales, históricas y sociales particulares. El "cuerpo sexuado" no es, en suma, para Butler más que "una herencia de actos sedimentados" (1988: 523), un "estilo corporal" (1988: 521).

\section{La sexualidad como "expresión" y como "estilo corporal"}

En la lectura que propondré a continuación, sin embargo, la teoría merleau-pontyana de la sexualidad como "expresión" no implica una concepción naturalista o esencialista como la que Butler le atribuye, sino que más bien y por el contrario supone una crítica de tales modelos. ${ }^{21}$ Para despejar este equívoco será necesario precisar en qué sentido particular afirma Phénoménologie que el cuerpo y la sexualidad son "expresiones", y además, qué sería esa "existencia" que "se expresa" en ellos.

En primer lugar, al afirmar que el cuerpo expresa la existencia, precisa Merleau-Ponty, hay que entender que "lo expresado no existe aparte de la expresión" sino que "los mismos signos inducen su sentido al exterior. Es de esta forma que el cuerpo expresa la existencia total, no porque sea su acompañamiento exterior, sino porque ésta se realiza en él" (1967: 193). La expresión así comprendida "no traduce al exterior un "estado interior', no constituye una 'manifestación"” (188), sino que el cuerpo, entendido como comportamiento, "es lo que significa" (188), su significación es "inmanente" (71) o "adherente" (172). La expresión no podría ser la exteriorización de un yo "interior", psicológico o intelectual, si ya el "Avant-Propos" a la Phénoménologie afirmaba que "no hay hombre interior, el hombre está en el mundo, es en el mundo que se conoce" (1967: v). Tampoco podría ser expresión de un yo permanente o estable previo que se expresara secundariamente en sus actos: por el contrario, Merleau-Ponty culmina su obra citando las palabras de Saint-Exupéry que afirman que "tú te alojas en tu acto mismo. Tu acto es tú" (520); "no es verdad que mi existencia se posea ni tampoco es verdad que sea ajena a sí misma, porque es un acto o un hacer" (438). No podría tampoco ser una mera instanciación individual de un bagaje biológico, orgánico o instintivo, puesto que "la dotación psico-fisiológica deja abiertas innumerables posibilidades y no hay aquí, como tampoco en el dominio de los instintos, una naturaleza humana dada de una vez por todas" (220).

¿Qué podría ser entonces esa "existencia" o esa "vida" que nuestra sexualidad "expresa" de modo inmanente? No es casual que Merleau-Ponty se refiera tres veces a la sexualidad como una "atmósfera" en el capítulo que le dedica, y retome la metáfora cerca del final de la obra para aludir a una "atmósfera de ‘socialidad" (511) inseparable del yo, que hace que "yo me capte desde el principio como excéntrico a mí mismo [...]

21 Coincidiré en este punto con Stoller cuando afirma que "la teoría de Butler de la performatividad y la teoría de Merleau-Ponty de la expresión comparten el rechazo a las teorías esencialistas" (2010: 109), si bien propondré a continuación una interpretación un tanto diferente de la noción merleau-pontyana de "expresión", subrayando además ciertas tensiones entre ambxs autorxs más allá de la "compatibilidad" que la autora constata como última conclusión de sus análisis comparativos.

22 A la luz de estas explícitas afirmaciones de Phénoménologie, se hace evidente también que resulta injustificable la interpretación de Butler que sostiene que el término "encarnación" (incarnation; embodiment) en Merleau-Ponty tiende a "mantener la relación externa y doble entre una inmaterialidad significante y la materialidad del cuerpo en sí" (Butler, 2010: 12, n. 15). 
[y] que mi vida tenga un sentido que yo no constituyo, que haya en rigor una intersubjetividad" (512). La sexualidad expresa para Merleau-Ponty la existencia en sus diversas dimensiones afectivas, psicológicas, y aun políticas, históricas y económicas -ejes a los que se refiere la extensa nota al pie con que culmina el capítulo sobre la sexualidad-, siendo todos ellos registros de carácter intersubjetivo o "intercorporal". Expresa, por lo tanto y en suma, nuestra fundamental coexistencia, nuestras relaciones con los otros en estos múltiples planos, relaciones que, además, nos constituyen precisamente como los que somos: "El hombre no es más que un nudo de relaciones" (Merleau-Ponty, 1967: 520). Butler capta perfectamente tanto el sentido propiamente merleau-pontyano de "expresión" como el de aquello que la sexualidad "expresa" en una obra muy posterior a aquel escrito en que parece malentenderlo, cuando afirma, en Undoing Gender, que la sexualidad no es un atributo ni una inclinación, sino "un modo de estar dispuestx hacia lxs otrxs":

nuestro ser al lado de nosotrxs mismxs, fuera de nosotrxs mismxs, es una función de la sexualidad misma, donde la sexualidad no es esta o aquella dimensión de la existencia, ni la clave, ni la base de nuestra existencia, sino más bien coextensiva con la existencia como Merleau-Ponty acertadamente sugirió (Butler, 2004: 33).

Llamativamente, los términos mismos con los que Butler define su teoría performativa del género como "estilo corporal" para contraponerla a la concepción merleau-pontyana, son términos tomados en préstamo de Merleau-Ponty. Ya observamos que el fenomenólogo cifra la clave de nuestra experiencia corporal y la correlativa experiencia del mundo y de lxs otrxs en la sedimentación de habitualidades de comportamiento, y Merleau-Ponty se refiere a ellos precisamente como "estilos" corporales de movimiento o comportamiento. Así habla, por ejemplo, de un "estilo de los gestos" (1967: 176) o de un "un estilo de conducta que mi cuerpo 'comprende'" (461). Los análisis acerca del hábito, concentrados en las últimas páginas del tercer capítulo de la Parte I de Phénoménologie, ya dejan traslucir, además, algo que las secciones posteriores de la obra referidas a la sexualidad, al lenguaje y al "otro" declaran frontalmente: el carácter intrínsecamente intersubjetivo o intercorporal y, por ende, histórico y cultural del esquema corporal. La observación merleau-pontyana de que "habituarse a un sombrero, a un coche o un bastón" es "hacerlos participar de la voluminosidad de nuestro cuerpo, [...] dilatar nuestro ser en el mundo, o cambiar la existencia anexándonos nuevos instrumentos" (1967: 168), supone que el esquema corporal se constituye mediante relaciones intercorporales pautadas en el contexto de comunidades situadas e históricas. Los instrumentos técnicos no son más que amplificaciones de nuestras capacidades sensoriales y motrices, y a su vez, nuestros hábitos motrices, por sí mismos, no son otra cosa que "técnicas del cuerpo" -en el sentido de M. Mauss (1934)- compartidas y perpetuadas en marcos sociales determinados, tan idiosincráticos, culturales e históricos como las estilográficas y las máquinas de escribir que menciona Merleau-Ponty en sus ejemplos: "El primero de los objetos culturales y aquel por el que existen todos es el cuerpo [...] como portador de un comportamiento" (1967: 401). ${ }^{24}$

Entre los ejemplos elegidos por Merleau-Ponty para analizar la adquisición de estas habitualidades constan precisamente algunos que caracterizan estereotipos de género de nuestra cultura: Merleau-Ponty se refiere al uso de un sombrero con pluma por parte de "una mujer" e, inmediatamente a continuación y como contrapartida, a la conducción de un automóvil por parte de un hombre (el autor enuncia en ese caso 
su descripción en primera persona) (1967: 167). Resulta improbable la interpretación de que Merleau-Ponty considere que los sombreros con pluma sean natural o esencialmente femeninos y los automóviles masculinos, sino más bien y por el contrario, el uso de los ejemplos invita fácilmente a pensar que los cuerpos generizados masculina o femeninamente son por sí mismos, aun en sus comportamientos y gestos no instrumentales, adquisiciones y artefactos culturales, tan culturales como lo son el sombrero y el automóvil que pasan a ser sus "partes" componentes por medio de la sedimentación de habitualidades. Esto es, en definitiva, lo que significa la definición merleau-pontyana del "cuerpo sexuado" no como anatomía ni como representación, sino como "esquema sexual": el cuerpo es "sexuado", tanto en el sentido de su deseo como de su identidad, de su orientación como de su género, en la medida en que cuenta con un repertorio de pautas de comportamiento corporal e intercorporal adquirido en contextos sociales particulares y sedimentado como un "estilo", pautas que definen el modo en que nos sentimos corporalmente generizadxs y "percibimos eróticamente" otros cuerpos.

En la línea de esta misma concepción de la sexualidad que ya proponía su Phénoménologie, en un ensayo posterior Merleau-Ponty escribe que "una mujer que pasa no es primeramente para mí un contorno corporal, un maniquí coloreado, un espectáculo", sino que es, en cambio:

una cierta manera de ser carne dada enteramente en el caminar o aun en el solo golpe del talón sobre el suelo [...] [;] una variación notable de la norma del caminar, del mirar, del tocar, del hablar que yo poseo en mí mismo porque soy cuerpo (1960: 67).

El párrafo no enuncia, como podría entenderse rápida y equívocamente, que el hombre sea esencialmente el sujeto y la norma universal, y la mujer el objeto y una variación de la norma masculina. Por el contrario, un autor que explicita su posición subjetiva de hombre viendo a una mujer afirma que, a despecho de la situación particular que su descripción toma como punto de partida, la mujer no es esencialmente un objeto visto ("un espectáculo"), lo que implica, correlativamente, que el hombre que ve no es esencialmente un sujeto vidente. Ambos, hombre y mujer, son así redefinidxs como modulaciones posibles del "ser cuerpo", maneras tipificadas de moverse. Desde esta perspectiva, la "norma" del sujeto masculino que ve resulta ser, en definitiva, solo una variedad posible de estas pautas estandarizadas del comportamiento corporal a mismo título que aquello que, desde su posición subjetiva particular (masculina), se le aparece como "variación".

\section{La teoría merleau-pontyana de la sexualidad redescubierta}

Es por haber comprendido en este preciso sentido la definición merleau-pontyana del cuerpo como "esquema corporal" que Beauvoir en Le deuxième sexe pudo recoger la afirmación de Phénoménologie que dice que "la humanidad no es una especie animal: es una realidad histórica" (Beauvoir, 1976a: 83), adaptando lo dicho por Merleau-Ponty acerca de la humanidad para referirse particularmente a la mujer. Así, la filósofa pudo afirmar que ningún destino anatómico determina lo que la mujer es sino que su ser resulta de una elaboración o un "llegar a ser" en el que interviene "el conjunto de la civilización" (1976b: 7). A su vez, tras leer Le deuxième sexe MerleauPonty ratificó su coincidencia con la visión de Beauvoir, sosteniendo en sus cursos 
de psicología que "no es posible hablar de lo femenino y de lo masculino puesto que cada civilización elabora un cierto tipo de masculinidad correlativo de un cierto tipo de feminidad [...]. Hay, en cambio, al interior de una sociedad dada, un estereotipo sexual" (Merleau-Ponty, 1988: 495). Estas afirmaciones, aun si posteriores, no suponen ningún giro respecto del punto de vista ya expuesto en Phénoménologie, si se recuerda que tal obra ya manifestaba que "incluso los [sentimientos] que, como la paternidad, parecen inscritos en el cuerpo humano son, en realidad, instituciones" (Merleau-Ponty, 1967: 220).

Numerosxs teóricxs posteriores reconocieron la singularidad de esta visión merleaupontyana de la sexualidad como esquema o estilo de comportamiento, en la medida en que resitúa la experiencia sexual en su enclave propiamente corporal -la sexualidad no es solo ni primeramente una cuestión psicológica, representacional o lingüística-, pero lo hace sin reducir el cuerpo a su definición anátomo-fisiológica: la sexualidad, como género y como deseo, es vivida materialmente en nuestros cuerpos, pero la materia del cuerpo tiene una historia. Y esta historia del cuerpo no es solo la de sus diversas representaciones científicas epocales o sus variables representaciones lingüísticas, sino, más profundamente, una historia propia y propiamente material: una historia social en la que los cuerpos son configurados diferentemente de acuerdo con las modalidades de comportamiento y percepción privilegiados por cada sociedad; una historia personal en la que los cuerpos son asumidos de distintas maneras en el curso de la vida; una historia, además, en la que los cuerpos mismos se conforman y transforman mediante la incorporación de prótesis técnicas, útiles, máquinas, vestimentas o vehículos que modifican y encauzan sus capacidades, así como espacios urbanos y arquitectónicos que regulan sus fórmulas de movimiento posible y pautan sus relaciones intercorporales de acuerdo a patrones culturales.

Un primer corolario que se desprende directamente de esta concepción es que ni la masculinidad ni la feminidad, resignificadas en estos términos, designan capacidades y roles fijos a cumplir en la organización familiar, laboral o política: retomando los términos ya citados del propio Merleau-Ponty, las variadas "masculinidades" y "feminidades" existentes en las distintas culturas hacen patente que estos diversos roles y factores pueden elaborarse y combinarse de distintos modos, incluso más allá de aquelllos que nos son ya conocidos en el pasado y el presente. En términos de Oksala, "cuando el cuerpo-sujeto de Merleau-Ponty no es entendido como universal ni fundacional sino como esencialmente dinámico e históricamente constituido, la implicancia para la teoría feminista es que no existen modos normales o fundacionales de corporeidad, motricidad o sexualidad femenina" (Oksala, 2006: 222). Beauvoir agregaría, más particular y enfáticamente, que "los datos biológicos" no constituyen para la mujer "un destino petrificado. No bastan para definir una jerarquía de los sexos; [...] no la condenan a conservar eternamente ese papel subordinado" particular que ha tenido y tiene aún en nuestra cultura sexista (1976: 67-68). ${ }^{26}$

En segundo lugar, y tal como lo ha puesto de relieve la recuperación de la fenomenología merleau-pontyana en el contexto de los estudios trans*, la propuesta de Merleau-Ponty sitúa la clave de la identidad de género no en factores anatómicos o fisiológicos innatos sino en la propiocepción -regida por el esquema corporal- y en el "estilo de movimiento" corporal, entendido en el sentido amplio de la gestualidad, el modo de hablar, de vestirse, etc. (cfr. Rubin, 1998; Salamon, 2014). El esquema corporal -y sexual- es un repertorio de capacidades de comportamiento que siento en 
mí mismx, y que determina el modo en que "asumo" o resignifico en la práctica mi cuerpo orgánico y mi cuerpo actual (con sus características actualmente perceptibles) (cfr. Merleau-Ponty, 1967: 97 ss.), definiendo así mi propiocepción y, particularmente en la esfera sexual, mi identidad de género autopercibida.

En tercer lugar, si bien esta teoría observa una correlación entre la propiocepción y la percepción del mundo o de lxs otrxs, lo que en este ámbito de análisis sugeriría que la identidad y el deseo están siempre de algún modo conectados, ella no implica que haya ningún modo fijo, universal ni natural que tal correlación deba adoptar. Es decir que, al contrario de lo que supone Butler, nada liga a una teoría de esta índole a una supuesta "heterosexualidad obligatoria" (cfr. supra, n. 14) sino que ella permite más bien pensar en una "variación sexual benigna" (cfr. Butler, 1989: 86): en palabras de Merleau-Ponty, el deseo otorga "valor o significación sexuales a los estímulos exteriores y design[a] para cada sujeto el uso que hará de su cuerpo objetivo" (1967:182).

En cuarto término, en la medida en que, de acuerdo con Merleau-Ponty, los estilos corporales son producto de la sedimentación habitual de actos pasados (adquiridos en contextos sociales) que son a la vez perpetuados y reasumidos en los actos personales actuales, su teoría establece una relación dialéctica abierta entre lo social y lo personal, así como entre el pasado y el presente personales. ${ }^{28}$ Acerca del primer aspecto, afirma Phénoménologie que "es imposible decir dónde terminan las fuerzas de la historia y dónde empiezan las nuestras [...] [;] no hay historia más que para un sujeto que la viva, y no hay sujeto más que históricamente situado" (201, n. 1). Y en torno al segundo aspecto, la obra sostiene que "la vida de la conciencia -vida cognoscente, vida del deseo o vida perceptiva-" deposita "los vestigios de sus propios actos, pero [...] se apoya en ellos para pasar a otros actos de espontaneidad" (158159). Este espacio de juego acordado a los actos de innovación que introducen lo inédito en la existencia, apoyándose y trascendiendo a la vez la tradición histórica y el pasado personal, deja abierto el camino para concebir y reconocer no solo otras feminidades y masculinidades -más allá de las que usan "el sombrero" y "el automóvil"- sino otras identidades posibles más allá de la diferencia sexual binaria, otros deseos más allá de la heterosexualidad o la homosexualidad, así como una sexualidad no fijada de una vez y para siempre en la vida personal sino abierta a una deriva (post-)identitaria y deseante, que no necesariamente encuentra un nombre o una categoría en los casilleros sociales disponibles, y fuerza a nuestro lenguaje y nuestro pensamiento adquiridos a hablar y pensar de maneras nuevas, a renovarse y cobrar vida (cfr. Ahmed, 2006; Burke, 2016; Lawlor, 2008; Oksala, 2006). El lenguaje hablado y el pensamiento pensado vuelven a ser hablantes y pensantes en contacto con la singularidad de nuestra vida, y no sería entonces extraño que, siendo la sexualidad un emblema de nuestra existencia, sea precisamente "en el trabajo paciente y silencioso del deseo [que] comience la paradoja de la expresión" (Merleau-Ponty, 1964: 189).

\section{Consideraciones finales: imaginando una teoría fenomenológico- performativa de la sexualidad}

A la luz de los análisis previos la teoría merleau-pontyana de la sexualidad como estilo corporal y como expresión de la existencia comprendida en su carácter intersubjetivo, situado e histórico se muestra tan alejada de una concepción naturalista y esencialista

27 El término "autopercepción" que utilizo intencionadamente acarrea en el contexto local (argentino) un valor particular, constando en la Ley № 26.743 (2012) que establece el derecho a que se reconozca la identidad de género "autopercibida" (arts. 1-3).

28 Como señala acertadamente Oksala, "en las descripciones de Merleau-Ponty [...] el cuerpo se muestra como promotor de reiteraciones tanto normativas como transgresoras", innovadoras y productivas (2006: 224). 
como la teoría performativa que Butler situaba en sus antípodas. Y, sin embargo, no deja de ser cierto que no podríamos leer hoy la Phénoménologie tal como propuse aquí leerla si no contáramos con las diversas perspectivas teóricas feministas que la redescubren desde problemas no explicitados por sí mismos en ella. Es por eso que, en este sentido y a pesar de todos los matices introducidos, las tempranas críticas de Butler a Phénoménologie conservan su validez intacta. Si en sus descripciones de la experiencia sexual Phénoménologie adopta en la enunciación el género masculino como universal, aun si fuera por seguir inercialmente los usos de la época, ¿no se arriesga inadvertidamente a masculinizar la descripción de la experiencia borrando las particularidades de la experiencia femenina? Esto es lo que advertía Beauvoir cuando decía, citando a Merleau-Ponty, que ciertamente "la mujer, como el hombre, es su cuerpo: pero su cuerpo es algo distinto de ella misma" (1976a: 60, n. 24). Es también lo que observa I. M. Young cuando señala que, si la sexuación se cifra en el "esquema sexual" como acervo de capacidades comportamentales -y esto lo vio Merleau-Ponty con exactitud- hay que agregar, continuando los análisis merleau-pontyanos pero yendo a la vez más allá de ellos, que "las mujeres en la sociedad sexista son discapacitadas físicamente" (Young, 2005: 42). Y aun si la adopción de Merleau-Ponty del punto de vista de un sujeto masculino respondiera a un estilo o un método fenomenológico, descriptivo y subjetivo de enunciación filosófica, sigue siendo cierto que el filósofo no advirtió que lo personal no solo es social e histórico sino también político. Dicho de otro modo, el autor de Phénoménologie no se percató de que la identificación del sujeto con el hombre, además de ser en este caso una marca genérica particular del fenomenólogo, es también y a la vez un ejercicio político generalizado en una sociedad patriarcal que despoja de ese rol al género femenino en la práctica, exclusión que, si el discurso y el pensamiento no explicitan, se arriesgan a perpetuar.

En suma y más profundamente, lo que Butler señala como una deuda o un límite propio de la propuesta merleau-pontyana -y este déficit es patente, lo que hace incontestable su crítica- es que no basta con afirmar que la sexualidad "expresa" las relaciones interhumanas tal como se dan en cierto contexto social e histórico, y es en ese sentido "construida" o "instituida", para usar un término merleau-pontyano: es necesario decir además que la sexualidad es "una construcción que oculta su génesis" (Butler, 1988: 522), que borra su carácter histórico y cultural para presentar como únicas e inalterables las relaciones de poder que la atraviesan y que ella también "expresa". No es suficiente afirmar, como efectivamente lo hace Merleau-Ponty, que la sexualidad no brota naturalmente desde un yo estable o un sustrato biológico puesto que es un estilo de comportamiento atado a patrones culturales, sino que hay que afirmar además que la repetición estereotipada de esos comportamientos -o la "repetición estilizada de actos", en términos de Butler- crea "la apariencia", "la ilusión" o "la creencia" en un yo, una identidad o una naturaleza inmutables de la cual los actos surgirían, ilusión que sirve precisamente para fijarlos y asegurar su reproducción (Butler, 1988: 519-520). No alcanza con manifestar que las categorías sexuales son instituciones sociales, sino que es necesario observar que son además instituciones normativas, asimétricas y jerárquicas que sancionan cotidianamente las desviaciones respecto de la norma de muy diversos modos, mediante la marginación, la criminalización, la patologización, la invisibilización, y aun la mutilación, el castigo y la violencia físicos. Es por ello que, si, como creo haber mostrado, la teoría merleau-pontyana de la sexualidad no solo es compatible sino que aportó y sigue aportando elementos claves para los posteriores estudios de género en clave feminista, hay que decir también, a la inversa, que es a

29 Ya se trate de mujeres que no cumplen el rol subordinado que se les asigna en la pareja, la familia o el trabajo, personas cuyo deseo no se ajusta al asignado culturalmente a su género, personas cuyo estilo corporal no es coherente con el que nuestra cultura asigna a su anatomía o bien no se ajusta al estilo corporal típico de uno de los dos géneros, personas cuyo cuerpo sexuado "varía respecto al standard de corporalidad femenina o masculina culturalmente vigente" (Cabral, 2005: 284), etc., etc. 
la luz de estos últimos que necesita ser redescubierta y releída para revelar tanto sus límites como su fecundidad en el contexto de las discusiones contemporáneas. En uno de aquellos tempranos ensayos en los que Butler criticaba rigurosamente a Merleau-Ponty, agregaba que, sin embargo, imaginaba claramente la posibilidad de desarrollar una "genealogía crítica del género que repose en un conjunto fenomenológico de presupuestos, especialmente la concepción ampliada de un 'acto', que es a la vez socialmente compartido e históricamente constituido, y performativo en el sentido más arriba descripto" (1998: 530). Imaginar una teoría de esa índole es una tarea ya iniciada desde hace décadas y que sigue abierta al pensamiento actual y al del futuro, y para cuyo desarrollo la Phénoménologie de la perception, a setenta y cinco años de su publicación, sigue revelando su utilidad y su vigencia. 


\section{Bibliografía}

"Ahmed, S. (2006). Queer Phenomenology. Orientations, Objects, Others. Durham: Duke University Press.

"Alcoff, L. M. (2000). Phenomenology, Post-structuralism, and Feminist Theory on the Concept of Experience. En Fisher, L.-Embree, L. (Eds.) (2000). Feminist Phenomenology (pp. 39-56). Dordrecht: Kluwer Academic Publishers.

»Burke, M. M. (2016). Love as a Hollow: Merleau-Ponty's Promise of Queer Love. Hypatia, X (10), 1-15.

" Beauvoir, S. de (1976a). Le deuxième sexe I. Les faits et les mythes (1949). París: Gallimard.

" Beauvoir, S. de (1976b). Le deuxième sexe II. L'expérience vécue (1949). París: Gallimard.

"Beauvoir, S. de (2007). El segundo sexo (trad. García Puente, J.). Buenos Aires: Sudamericana.

» Bolla, L. (2015). Butler lectora de Sartre. Las críticas al sujeto del deseo sartreano (desplazamientos y filiaciones). En Femenías, M. L.-Martínez, A. (coords.). Judith Butler. Las identidades del sujeto opaco (pp. 73-85). La Plata: Universidad Nacional de La Plata.

» Bordo, S. (1998). Bringing Body to Theory. En Welton, D. (Ed.) Body and Flesh: A Philosophical Reader (pp. 84-97). Oxford: Blackwell.

» Bourdieu, P. (1998). La domination masculine. París: Éditions du Seuil.

" Butler, J. (1987). Subjects of Desire. Hegelian Reflections in Twentieth-Century France. New York: Columbia University Press.

" Butler, J. (1988). Performative Acts and Gender Constitution: An Essay in Phenomenology and Feminist Theory. Theatre Journal, 40 (4), 519-531.

» Butler, J. (1989). Sexual Ideology and Phenomenological Description: A Feminist Critique of Merleau-Ponty's Phenomenology of Perception. En Allen, J.-Young, I. M. (Eds.). The Thinking Muse. Feminism and Modern French Philosophy (pp. 85-100). Bloomington: Indiana University Press.

" Butler, J. (2004). Undoing Gender. New York: Routledge.

» Butler, J. (2006a). Merleau-Ponty and the Touch of Malebranche. En Carman, T.Hansen, M. (Eds.). The Cambridge Companion to Merleau-Ponty (pp. 181-205). Cambridge: Cambridge University Press.

»Butler, J. (2006b). Sexual Difference as a Question of Ethics: Alterities of the Flesh in Irigaray and Merleau-Ponty. En Olkowski, D. - Weiss, G. (Eds.). Feminist Interpretations of M. Merleau-Ponty (pp. 107-126). Penssylvania: The Pennsylvania State University Press.

» Butler, J. (2010). Gender Trouble. Feminism and the Subversion of Identity (1990). New York: Routledge Classics.

" Butler, J. (2011). Bodies That Matter. On the discursive limits of "sex". London: Routledge Classics.

" Butler, J.-Cano, V.-Fernández Cordero, L. (2019). Vidas en lucha. Conversaciones. 
Buenos Aires: Katz.

» Cabral, M.-Benzur, G. (2005). Cuando digo intersex. Un diálogo introductorio a la intersexualidad. Cadernos Pagu, 24, 283-304.

»Chanter, T. (Ed.) (2001). Feminist Interpretations of Emmanuel Levinas. Pennsylvania: Pennsylvania State University.

" Elliston, F. A. (1981). Introduction to Universal Teleology. En Husserl. Shorter Works. Notre Dame: University of Notre Dame Press.

» Fisher, L. (2000). Phenomenology and Feminism. Perspectives on their Relation. En Fisher, L.-Embree, L. (Eds.). Feminist Phenomenology (pp. 17-38). Dordrecht: Kluwer Academic Publishers.

» Foultier, A. P. (2013). Language and the Gendered Body: Butler's Early Reading of Merleau-Ponty. Hypathia, 28 (4), 767-783.

» García, E. A. (2012). Merleau-Ponty en la reflexión contemporánea acerca de las políticas normativas del cuerpo. En Ramírez, M. T. (Coord.). Merleau-Ponty viviente (pp. 345-370). Barcelona, Anthropos Editorial.

»Haraway, D. (1991). Simians, Cyborgs and Women. The Reinvention of Nature. New York: Routledge.

» Heinämaa, S. (2003). Toward a Phenomenology of Sexual Difference. Husserl, Merleau-Ponty, Beauvoir. Maryland: Rowman \& Littlefield Publishers.

» Husserl, E. (1973). Husserliana XV. Zur Phänomenologie der Intersubjektivität. Texte aus dem Nachlass. Dritter Teil: 1929-1935. Den Haag: Martinus Nijhoff.

"Husserl, E. (1954). Husserliana VI. Die Krisis der europäischen Wissenschaften und die tranzendentale Phänomenologie, eine Einleitung in die phänomenologische Philosophie. La Haye: Martinus Nijhoff, 1954.

» Lawlor, L. (2008). Benign Sexual Variation: An Essay on the Late Thought of Merleau-Ponty. Chiasmi International, 10, 47-56.

» Levinas, E. (1990). De l'existence à l'existant (1947). París: Vrin.

» Mauss. M. (1936). Les techniques du corps. Journal de Psychologie, XXXII (3-4).

» Merleau-Ponty, M. (1960). Signes. París: Gallimard.

» Merleau-Ponty, M. (1964). Le Visible et l'Invisible. Suivi de notes de travail par M. Merleau-Ponty. París: Gallimard.

» Merleau-Ponty, M. (1967). Phénoménologie de la perception (1945). París: Gallimard.

" Merleau-Ponty, M. (1988). Merleau-Ponty à la Sorbonne. Resumé de cours 19421952. Dijon-Quetigny: Cynara.

» Oksala, J. (2004). What is feminist phenomenology? Thinking birth philosophically. Radical Philosophy, 126, 16-22.

» Oksala, J. (2006). Female Freedom: Can the Lived Body Be Emancipated? En Olkowski, D.-Weiss, G. (Eds.). Feminist Interpretations of M. Merleau-Ponty (pp. 209-228). Penssylvania: The Pennsylvania State University Press.

» Palacio, M. (2011). La diferencia sexual en el pensamiento de E. Levinas. Isegoría. Revista de Filosofía Moral y Política, 45, 669-682.

» Rubin, H. (1998). Phenomenology as Method in Trans Studies. GLQ. A Journal of Lesbian and Gay Studies, 4 (2), 263-281. 
» Rojcewicz, R.-Schuwer, A. (1989). Translator's Introduction. En Husserl, E. Ideas Pertaining to a Pure Phenomenology and to a Phenomenological Philosophy. Second Book: Studies in the Phenomenology of Constitution. Dordrecht: Kluwer Academic Publishers.

» Salamon, G. (2014). Phenomenology. TSQ. Transgender Studies Quarterly, 1 (1-2), 153-155.

» Sartre, J.-P. (1940). L'Imaginaire: Psychologie phénoménologique de l'imagination. París: Gallimard.

» Sartre, J.-P. (1943). L'être et le néant. Essai d'ontologie phénoménologique. París: Gallimard.

»Stoller, S. (2010). Expressivity and Performativity: Merleau-Ponty and Butler. Continental Philosophical Review, 43, 97-110.

"Young, I. M. (2005). Throwing Like a Girl. A Phenomenology of Feminine Body Comportment, Motility, and Spatiality. En On Female Body Experience. "Throwing Like a Girl" and Other Essays. New York: Oxford University Press. 